\title{
LOS PROCESOS DE ADAPTACIÓN AL CAMBIO CLIMÁTICO EN ESPACIOS INSULARES: EL CASO DE CANARIAS
}

\author{
Abel LÓPEZ DÍEZ ${ }^{1}$, Pedro DORTA ANTEQUERA, Miguel FEBLES RAMÍREZ², \\ Jaime DÍAZ PACHECO ${ }^{1}$ \\ ${ }^{1}$ Universidad de La Laguna. Cátedra "RRD. Ciudades Resilientes". \\ ${ }^{2}$ GEODOS, Planificación y Servicios S.L. \\ alopezd@ull.es, pdorta@ull.es, miguelfebles@geodos.es, jdiazpac@ull.es
}

\section{RESUMEN}

Los efectos del cambio climático constituyen uno de los principales problemas a los que se enfrenta la sociedad. Cada vez son más las evidencias científicas que los constatan, manifestándose a través de algunas variables climáticas, especialmente las temperaturas. Estos cambios que se están produciendo en la actualidad son la antesala de un proceso que se desarrollará a medio y largo plazo, y cuyos impactos en sectores de actividad como el turismo pueden suponer un gran condicionante para economías locales. Los mencionados efectos están, por tanto, requiriendo la implementación de acciones de adaptación territorial al cambio climático.

A través de una exploración de antecedentes se exponen los principales estudios vinculados con el cambio climático, en este caso enfocados a una región insular atlántica, como las Islas Canarias que, además, presentan una gran complejidad en términos económicos y sociales. En este sentido, este estudio, no sólo revisa aquellas cuestiones relacionadas con las variaciones ambientales producidas por el cambio climático, sino que además realiza un análisis de las diferentes medidas de adaptación que hasta ahora han sido efectuadas en dichas islas.

Palabras clave: adaptación, cambio climático, Islas Canarias, vulnerabilidad.

\begin{abstract}
The climate change effects are one of the major problems that the society is currently facing. The scientific evidences observed through climatic variations, specially the increasing of temperatures, are already proving it. These changes are occurring at present and they are the prelude to a process that will be developed at medium and long term, and whose impacts on sectors such as tourism can be a major constraint for local economies. The aforementioned effects are therefore, requiring the implementation of actions for land adaption to climate change. Through an exploration of history major studies are discussed with relation to the climate change, and in this case, focused on an Atlantic island region, the Canary Islands. These islands present a huge complexity in economic and social terms.

In this regard, this study not only reviews questions relating to environmental variations caused by climate change, but also an analysis of different adaptation measures that have so far been implemented in these islands.
\end{abstract}

Key words: adaptation, climate change, Canary Islands, vulnerability. 


\section{INTRODUCCIÓN}

Cada vez son más las evidencias científicas que constatan un calentamiento del sistema climático, sirva de referencia que el año 2015 fue el más caluroso desde que se registran datos de temperatura, o la serie de siete meses consecutivos hasta abril de 2016 batiendo récords de temperatura media planetaria. Todo ello hace prever que 2016 será aún más cálido que su predecesor. Asimismo, este progresivo calentamiento tiene como su principal causa la injerencia humana sobre el sistema natural, tal y como evidencia el Quinto Informe del IPCC (2013). En este contexto, se hace necesario conocer los posibles riesgos y efectos derivados del cambio climático con el fin de adoptar las medidas y respuestas necesarias enfocadas a la mitigación y adaptación ante este fenómeno. Mientras la mitigación está enfocada a la reducción de los gases efecto invernadero, la adaptación constituye el proceso de ajuste de la sociedad a las variaciones climáticas tanto actuales como futuras. Este hecho provoca que la adaptación sea un elemento clave a tener en cuenta en la planificación económica y territorial de una región.

El cambio climático pese a ser un problema global, debe entenderse como una cuestión multiescalar y multisectorial, aspecto éste que se encuentra reflejado en los documentos de consenso internacional, que se han desarrollado de una manera creciente en las últimas décadas hasta la actualidad; véase, por ejemplo, el reciente Acuerdo de París o el Marco de Sendai para la Reducción de Riesgos de Desastres, ambos de 2015. Estos documentos plantean cuestiones globales y propuestas de acción que suelen estar dirigidas a los países pero, en última estancia, son las entidades locales las que, en gran medida, deben implementar acciones de mitigación y adaptación de manera directa. En este sentido, dichas entidades locales empiezan a demandar marcos de actuación, herramientas, medios e instrumentos, para abordar y tratar las cuestiones asociadas al cambio climático donde, además, suelen ser más débiles e incompletos (Held, 2012). Desde una perspectiva multisectorial las respuestas frente al cambio climático han de integrar a los diferentes actores sociales y económicos, tanto privados como públicos siendo, esto último, esencial para que los procesos de adaptación y mitigación sean efectivos.

Los espacios insulares poseen una gran fragilidad ambiental, social y económica, pudiendo presentar alteraciones significativas ante el cambio climático. La transformación de estos aspectos en el caso de la región macaronésica y, en especial, de Canarias, supone transformaciones tanto en las condiciones climáticas como en el incremento de los riesgos territoriales, derivados de un previsible aumento de la peligrosidad ante determinados fenómenos meteorológicos. De esta manera, algunos de los efectos constatados para las Islas Canarias pueden amenazar a la principal actividad económica de las islas, el turismo. Una actividad que en un escenario de cambio climático futuro debe adaptarse si quiere mantener la actual competitividad (Olcina, 2012).

La adaptación ante el cambio climático es específica del lugar y del contexto de un territorio, por ello es imprescindible el conocimiento y desarrollo de bases científicas a escalas de mayor precisión espacial que permitan determinar con exactitud los posibles efectos e impactos asociados. De este modo, en este trabajo, se presenta, en una primera parte, un estudio acerca de las principales evidencias científicas consta- 
tadas que están afectando a una región específica, la Macaronesia. Luego se exploran los mencionados marcos, instrumentos, herramientas y medidas de adaptación que se están llevado a cabo en una entidad geográfica dentro la citada región, como son las Islas Canarias. Finalmente, se expondrán una serie de conclusiones que procuran construir un escenario que permita vislumbrar el punto en el que se encuentra una región insular, en lo que se refiere al conocimiento de los efectos territoriales del cambio climático y de las medidas implementadas en cuanto a la adaptación.

\section{FUENTES Y MÉTODO}

El siguiente trabajo aborda un estudio de los principales antecedentes sobre cambio climático en la región macaronésica. Para ello se ha recurrido a un profundo análisis de revisión bibliográfica, analizando dos líneas subsecuentes que interaccionan a diferentes escalas:

1. Las consecuencias globales del cambio climático y las medidas de adaptación propuestas a través de paneles intergubernamentales.

2. Los efectos locales del cambio climático y la adopción de medidas concretas de adaptación, tomando como caso un espacio insular.

Dentro de la escala local, se realiza una exploración de referencias científicas vinculadas con el cambio climático observadas en la región macaronésica. Por un lado, para reconocer en qué grado las manifestaciones del cambio climático están teniendo lugar en la región, y qué efectos derivados del calentamiento global comienzan a ser corroborados científicamente. Posteriormente, se examina, a través de publicaciones e informes instituciones, cuál es el desarrollo de medidas de adaptación al cambio climático que se han realizado a escala internacional, nacional y regional.

\section{CONTEXTO GEOGRÁFiCO}

El Atlántico norte suroriental es un espacio geográfico de gran interés climático por situarse en una región de transición entre la circulación templada y la tropical. La región de la Macaronesia engloba cinco archipiélagos, pertenecientes a tres países, y la integran más de 40 islas e islotes, además de territorios de la costa africana subtropical. Las islas macaronésicas presentan similitud geológica y biogeográfica, de origen volcánico y relieve complejo, comparten tipos de flora y fauna adaptada a condiciones climáticas comunes, con una alta biodiversidad. En este sentido, los espacios insulares exhiben un gran interés como laboratorios en cuanto a la mitigación y adaptación frente al cambio climático (Tomé, et al., 2014) y, Canarias en particular, supone un excelente observatorio natural para el análisis de variabilidad en los patrones de circulación para el Atlántico Norte (García Herrera, et al., 2001). No obstante, es una región no bien conocida por la ausencia o carencia significativa de información meteorológica.

Los principales rasgos climáticos del archipiélago canario quedan definidos por su posición latitudinal subtropical y su situación en el Atlántico oriental, muy cerca de la costa africana y del desierto más cálido y extenso del planeta, el Sáhara. Térmicamente las islas presentan valores medios muy suaves por la presencia de la corriente fría de Canarias. Pluviométricamente un total de precipitaciones, en general, muy 
modesto por la estabilidad atmosférica predominante. Sin embargo, la presencia de un complejo y vigoroso relieve, sobre todo en las cinco islas más occidentales, crea importantes discontinuidades térmicas y pluviométricas. Esto, unido a la proximidad del Sáhara y a la llegada de depresiones templadas, en ocasiones profundas y muy bajas en latitud puede generar extremos atmosféricos muy marcados. Así, los valores tanto de las temperaturas máximas extremas como las máximas precipitaciones diarias son similares a los registrados en la Península Ibérica.

Al mismo tiempo, Canarias es uno de los territorio de mayor densidad demográfica no sólo de España, sino de toda Europa. Las dos islas centrales, Tenerife y Gran Canaria, poseen valores superiores a los 400 y $500 \mathrm{hab} / \mathrm{km}^{2}$ respectivamente. Además, a los más de 2 millones de residentes, se añaden todos los años más de 13 millones de turistas, asentados casi en su totalidad, en la franja costera. Toda esta población depende energéticamente del petróleo, que supone más del 95\% de la producción eléctrica en las islas. Por todo lo expuesto, desde una perspectiva socioeconómica, Canarias se muestra como un territorio de una elevada vulnerabilidad frente al calentamiento global, entendido en un sentido amplio, tanto en lo que se refiere a mitigación como, y sobre todo, a adaptación.

\section{EL CAMBIO CLIMÁTICO EN LA REGIÓN MACARONÉSICA}

Los sucesivos informes del IPCC han señalado durante los últimos 25 años el alcance y la evolución del calentamiento global. Los datos en el Atlántico Norte suroriental, en el que se encuentra la región macaronésica, evidencian también que este sector no es ajeno al mismo. La temperatura, sobre todo, y la precipitación, en menor medida, son los elementos climáticos más estudiados. En cuanto a las temperaturas, ya diversos trabajos científicos afirman su ascenso generalizado (Martín et al., 2012; Luque, et al., 2014, Cropper, 2013, Cropper and Hanna, 2013), especialmente pronunciado desde mediados de los años 70 y más significativo en las temperaturas nocturnas y la alta montaña, tal y como ocurre en otros espacios insulares similares (Díaz et al., 2011). Además, los análisis estadísticos de tendencia pluviométrica, aunque mucho más complejos en esta región, debido a la gran irregularidad de las precipitaciones y a la variabilidad topográfica, comienzan a sugerir un cambio en los patrones de circulación y una disminución de los totales pluviométricos con algo más de relevancia durante el otoño (Mayer et al., 2015). Se debe tener en cuenta que Canarias posee altísimos coeficientes de variación e índices de concentración de la precipitación (Máyer y Marzol, 2014). Aún así son varios los trabajos publicados al respecto que analizan diversos aspectos en las tendencias o con relación a fenómenos de teleconexión climática como la NAO (García-Herrera et al., 2003; Máyer et al., 2015; Tarife et al., 2012).

En este sentido, sobre el comportamiento de la presión se ha analizado la relación entre la NAO y las condiciones climáticas sobre el archipiélago (García Herrera, et al, 2000; Cropper and Hanna, 2013) e indirectamente, la circulación de los vientos alisios. Otros aspectos tratados son el ascenso del nivel del mar (Fraile Jurado et al., 2014) y el cambio en la temperatura superficial del mismo. En ambos casos con tendencias positivas, como en el resto del planeta, donde se ha estimado un ascenso de $0,19 \mathrm{~m}$ durante el periodo 1901-2010 (IPCC, 2013). Uno de los principales condicionantes 
climáticos del ámbito macaronésico es la corriente fría de canarias, cuya modificación asociada al calentamiento puede derivar en alteraciones climáticas significativas, tanto en la intensificación o debilitamiento de la misma como en la variación general del patrón de vientos dominantes (Semedo et al.,2016).

En los últimos años se han sucedido una serie de eventos graves en cuanto a daños y víctimas, provocados por la aparición de fenómenos meteorológicos extremos, algunos de los cuales podrían estar influenciados por el calentamiento global. Es el caso de varias olas de calor con valores térmicos que han superado ampliamente los máximos registrados hasta la fecha o precipitaciones de gran intensidad horaria (Dorta, 2007, López et al., 2015), con extremos que han llegado, incluso, a duplicar los máximos registrados y a superar periodos de retorno de más de 500 años. Además, se debe tener en cuenta que al Oeste de Cabo Verde se haya una región donde se originan las tormentas y ciclones tropicales más intensos que afectan al Caribe y a América del Norte (Cropper, 2013). Aunque es cierto que sólo muy puntualmente estos se han acercado a la Macaronesia, recientemente, algunos de ellos ha seguido trayectorias centradas en el Atlántico oriental afectando a los archipiélagos de Azores, Maderia y Canarias, como son los casos de Delta en otoño de 2005 o Nadine en otoño de 2012 (Brown, 2013). No se debe olvidar, en este sentido, que las proyecciones presentadas por el IPCC (2013) inciden en la alta probabilidad de que se intensifiquen los fenómenos meteorológicos extremos con el incremento de la temperatura atmosférica y oceánica.

\section{LA ADAPTACIÓN AL CAMBIO CLIMÁTICO}

Han sido múltiples los hitos en la reciente historia vinculados a las políticas medioambientales y el clima, desde el inicio con el Protocolo de Montreal (1989), la Convección Marco de las Naciones sobre el Cambio Climático (1992), el Protocolo de Kyoto adoptado en 1997 o el Acuerdo de Copenhague (2009). Sin embargo, las políticas vinculadas con la adaptación han ido desarrollándose de forma lenta aunque constante hasta la actualidad. Uno de los referentes en el desarrollo de la adaptación, se deriva de la COP11 (2005) en Montreal con el "Programa de trabajo quinquenal sobre los aspectos científicos, técnicos y socioeconómicos de los efectos, la vulnerabilidad y la adaptación al cambio climático" asentando las bases para la implantación de los procesos de adaptación a todas las escalas y definiendo unos resultados y programas de trabajo, siendo el precursor mediante el cual se desarrollará el Plan Nacional de Adaptación al Cambio Climático de España (PNACC). No obstante, no será hasta la celebración de la COP13 (2007), cuando las políticas de adaptación empiecen a tener mayor protagonismo a través de la "Hoja de Ruta de Bali" donde se establece que los procesos de negociación vinculados con la CMNUCC deberán centrarse en cuatro pilares, uno de ellos la adaptación. Con la COP16 en 2010 y el desarrollo del "Marco de Adaptación de Cancún", se crea el "Comité de Adaptación", que pretende impulsar todas aquellas acciones destinadas a promover la adaptación, como por ejemplo, desarrollando planes nacionales de adaptación. Este Marco establece un punto de inflexión entre las políticas de mitigación y adaptación, donde estas últimas toman una mayor importancia por primera vez. 
Los esfuerzos hasta la fecha para lograr la estabilización y reducción de las emisiones de GEI para evitar interferencias en el sistema climático han sido poco eficientes, como se evidencia con la publicación del Quinto Informe del IPCC. Por tanto, cada vez más, se hace más necesaria la adopción de medidas enfocadas, sobre todo, a la adaptación. Ante esta situación y mediante la aprobación del Acuerdo de París, el 12 de diciembre de 2015 en la COP21, se genera el documento marco que sustituye al protocolo de Kioto y que define los objetivos a nivel mundial para dar una respuesta eficaz al cambio climático, donde la adaptación adquiere mayor importancia (Art. 2,6,7,9). En el mismo ámbito internacional, recientemente se adoptó el "Marco de Sendai para la Reducción de Riesgos de Desastres" por la Oficina para la Reducción del Riesgo de Desastres de Naciones Unidas (UNISDR, 2015) un instrumento que incorpora el cambio climático como un factor desencadenante del riesgo de desastres, especificado de manera directa en sus cuatro prioridades. Asimismo, se determina la adaptación al cambio climático como una pieza básica en las medidas para reducir de forma eficaz los riesgos tanto directos como indirectos del calentamiento global.

Uno de los referentes dentro del ámbito internacional son los reportes del IPCC sobre adaptación al cambio climático, desarrollados desde el segundo de los informes publicado en 1995, hasta el último de 2014 “Impactos, Adaptación, y Vulnerabilidad", definiendo los principales efectos vinculados con el cambio climático y las medidas a implementar según los contextos espaciales y los riesgos esperados.

En Europa las políticas de acción sobre el cambio climático han ido dirigidas, casi en su totalidad, a la mitigación. Un buen indicador de ello son las numerosas directivas y decisiones encaminadas a reducir y controlar los GEI, así como a implementar el uso de energías limpias. De este modo, los procesos de adaptación a nivel europeo no se impulsan hasta 2009 con el libro blanco "Adaptación al cambio climático: Hacia un marco europeo de actuación", donde se establecen, por primera vez, unos objetivos y medidas a nivel comunitario para el estímulo de la adaptación. Posteriormente, en 2010, con la creación de la Dirección General de Acción contra el Clima, la adaptación comienza a desarrollarse de una manera más fehaciente. Este ente se encarga entonces de generar documentación como "La estrategia europea de adaptación al cambio climático". Con todo ello, las políticas europeas vinculadas con el cambio climático siguen, en la actualidad, centralizadas en la mitigación, a través de la confección de un marco normativo encaminado a la reducción de los gases de efecto invernadero y el control del agujero de ozono.

En España, tanto el Ministerio de Agricultura, Alimentación y Medio Ambiente (MAGRAMA) como el Ministerio de Industria, Energía y Turismo (MIEyT) son los competentes en la materia. De estos ministerios dependen los principales organismos destinados al desarrollo de acciones frente al cambio climático. Con la creación en 1992 de la Comisión Nacional del Clima se genera el primer órgano nacional destinado al estudio, asesoramiento e implementación del cambio climático. Esta Comisión fue sustituida en 1998 por el actual Consejo Nacional del Clima, cuya función es elaborar propuestas y recomendaciones sobre políticas relativas al cambio climático, incluidas las de adaptación. En 2001, se crea la Oficina Española del Cambio Climático (OECC), siendo el principal organismo destinado al desarrollo de las acciones vinculadas con el cambio climático. Es a partir de su creación cuando se efectúan las primeras acciones sobre adaptación en España, como la elaboración en 2006 del 
Plan Nacional de Adaptación al Cambio Climático (PNACC). La OECC trata de integrar las políticas de adaptación general en políticas sectoriales. En este contexto se aprueban normas nacionales donde se empiezan a integrar aspectos de mitigación y la adaptación al calentamiento global, como es el caso de la Ley 21/2013, de evaluación ambiental o el muy reciente Real Decreto Legislativo 7/2015 de la Ley de Suelo y Rehabilitación Urbana.

A escala nacional existen más organismos destinados de una forma directa e indirecta al desarrollo de acciones vinculadas con el cambio climático, como la Comisión de Coordinación de Políticas de Cambio Climático o la Comisión Nacional de Energía entre otros. De la misma forma, cabe destacar que se han desarrollado acciones con implicaciones en la adaptación al cambio climático como en 2007 la "Estrategia Española de Cambio Climático y Energía Limpia", o la "Estrategia local de cambio climático" elaborada por la Red Española de Ciudades por el Clima.

\section{LA ADAPTACIÓN EN LAS ISLAS CANARIAS}

En el de la Comunidad Autónoma de Canarias, el eje central de la lucha institucional contra el cambio climático es la "Estrategia Canaria de Lucha contra el Cambio Climático" (ECLCC), desarrollada por el Gobierno de Canarias (Resolución 7L/ CG-0010). En esta el Parlamento de Canarias insta al Gobierno de Canarias a implementar las medidas incluidas en la ECLCC, como la creación de la Agencia Canaria de Desarrollo Sostenible y Cambio Climático (ACDSCC). La ECLCC aprobada en el año 2009, tiene como pilares fundamentales un plan de mitigación, organizado y estructurado desde la propia ECLCC y un plan de adaptación, definiendo los criterios para su desarrollo posterior. Se debe resaltar la alusión expresa que realiza la ECLCC a la relación directa entre desarrollo sostenible y lucha contra el cambio climático, dando continuidad al esfuerzo legislativo realizado a principios de la década de los años 2000 con la aprobación de las Directrices de Ordenación General y Directrices de Ordenación del Turismo de Canarias (Ley 19/2003).

El objetivo del Plan de Mitigación es la reducción de GEI para los años 2010 y 2015, una disminución dirigida a determinados sectores estratégicos a través de un conjunto de medidas de carácter horizontal. Muchas de estas medidas son retomadas, tanto de documentos legales, como las Directrices de Ordenación del Territorio, como de otros Planes ya aprobados y que desarrollaban, en mayor o menor medida, acciones enfocadas a la mitigación, como es, entre otros, el Plan Energético de Canarias 2006-2015. El Plan de Mitigación, tenía previsto como instrumentos de seguimiento y evaluación por un lado el desarrollo de un inventario canario de emisiones de gases de efecto invernadero y la revisión, cada cuatro años, de la propia ECLCC. En 2008 se elaboró el primer y único inventario de GEI, resultado de un trabajo de análisis de información, así como de consulta con las Administraciones competentes y de aportaciones por entidades privadas, en particular las pertenecientes al sector industrial; dentro del marco del "Proyecto Clima Impacto".

Para la finalización de la ECLCC, así como para el desarrollo instrumental de la misma, se constituyó en el 2009 la ACDSCC. En el preámbulo de la ley se reconoce la complejidad de las tareas que ésta debe abordar, así como el necesario cambio de cultura, de políticas y formas de actuar. En este escenario se justifica que el menciona- 
do organismo tenga carácter autónomo, dotándola de cierta relevancia y un marcado carácter transversal. Entre sus funciones no sólo se encontraba la redacción de la ECLCC, sino también liderar el proceso de redacción de los planes de actuación y otros instrumentos de orientación, coordinación, planificación y programación del proceso, incluyendo el seguimiento a través de la elaboración de indicadores y el desarrollo de un sistema de información que sirva como base para la formulación de un diagnóstico ambiental de Canarias cada tres años. Esta Agencia es la que comienza los trabajos relativos a la implementación del "Plan de Adaptación al Cambio Climático". El desarrollo de este instrumento se realiza a partir de un conjunto de estudios previos en el periodo 2009-2011, quedando esbozado un documento que recoge una selección de medidas, métodos de trabajo, herramientas, así como los organismos y sectores con responsabilidad para llevar a cabo las medidas propuestas. El documento no finalizado, planteaba la necesidad del desarrollo sectorial del plan, a través de tres ejes; la implantación de medidas, la integración de la adaptación en las políticas de la Comunidad y un plan económico de las acciones de adaptación.

Este plan planteaba la incorporación de otras iniciativas ya existentes como el Foro Canario para el Desarrollo Sostenible, cuyo funcionamiento estaba previsto a través de comisiones, entre las que se encontraba una con exclusiva dedicación al Cambio Climático; o el Observatorio del Desarrollo Sostenible, como instrumento científico y técnico de evaluación y seguimiento de las políticas, planes y programas de desarrollo sostenible en el Archipiélago; ambas bajo el paraguas administrativo de la ACDSCC.

El desarrollo de la ECLCC queda paralizado en 2011. La ACDSCC se encuentra entre el conjunto de entes públicos que desaparecen entre 2011 y 2015 en el marco de las medidas adoptadas por el Gobierno de Canarias para reordenar y racionalizar el sector público autonómico y cumplir con los objetivos de estabilidad presupuestaria.

En la actualidad se está procediendo a una revisión profunda de la legislación sobre ordenación del territorio en Canarias, fundamentado en el Anteproyecto de Ley del Suelo de Canarias que tiene como principio fundamental de desarrollo sostenible. Este Anteproyecto de Ley que establece la derogación de gran parte de las directrices de la Ley 19/2003, tendrá consecuencias directas sobre la ECLCC, así como sobre sus dos pilares fundamentales: Plan de Mitigación y Plan de Adaptación al Cambio Climático.

Realizado un repaso por el marco normativo de canarias sobre el gobierno del cambio climático, hay que señalar que en los últimos años se han implementado iniciativas vinculadas indirectamente con el el cambio climático y la adaptación, tales como la adhesión de 78 de los 88 municipios de Canarias a la campaña "Desarrollando Ciudades Resilientes" de UNISDR o la inclusión de instituciones como el Cabildo de Tenerife y varios municipios del archipiélago en el denominado "Pacto de los alcaldes para el clima y la energía."

\section{CONCLUSIONES}

El cambio climático es uno de los mayores problemas territoriales a los que se enfrenta la sociedad. Cada vez son más las evidencias científicas que corroboran variaciones en el sistema climático y la región macaronésica no es una excepción: ya se 
empiezan a evidenciar cambios significativos en determinados elementos del sistema climático, como las temperaturas o el régimen pluviométrico.

Tanto a escala internacional como nacional se está generando un amplio marco de normas y recomendaciones asociadas con la adaptación. Nuevas normas nacionales, como la Ley de Evaluación Ambiental, incorporan ya análisis de planes y proyectos relativos a la adaptación al cambio climático. Sin embargo, se observa la existencia de un vacío metodológico a la hora de llevar a cabo estos análisis. Las Islas Canarias han centralizado en la ECLCC las acciones asociadas con el cambio climático. La Agencia Canaria de Desarrollo Sostenible y Cambio Climático ha sido el ente encargado de elaborar los planes de mitigación y adaptación, unos planes cuyo desarrollo quedo paralizado en 2011, hecho que ha provocado que apenas se hayan impulsado políticas de adaptación al cambio climático en las islas más allá de las acciones ya comentadas. Todo ello, en un contexto territorial que presenta una gran vulnerabilidad y exposición a los efectos y riesgos derivados del cambio climático. A través de este estudio se concluye que se requiere de grandes esfuerzos que permitan implementar las mencionadas acciones y, sobre todo, que estén vinculadas a procesos de alcance basados en participación e integración de todos los agentes. Esto, en última instancia es lo que permitirá desarrollar mejores sistemas de gobernanza local proactiva respecto al cambio climático.

\section{REFERENCIAS}

Brown, D. (2013). Tropical Cyclone Report Hurricane Nadine. National Hurricane Center.

Cropper, T.E. (2013). The weather and climate of Macaronesia: past, present and future. Weather. Vol.68, N.11, pp. 300-307. doi: http://dx.doi.org/10.1002/wea.2155

Cropper, T. E., Hanna, E. (2013). An analysis of the climate of Macaronesia, 1865-2012. International Journal of Climatology. Vol. 34, N. 6, pp. 604-622.

Diaz, F., Giambelluca, T., Eischeid, J. (2011). Changes in the vertical profiles of mean temperature and humidity in the Hawaiian Islands. Global and Planetary Change, Vol. 77, N.1, pp. 21-25.

Dorta, P. (2007). Catálogo de riesgos climáticos en Canarias: amenazas y vulnerabilidad. Geographicalia, Vol. 51, pp. 33-160.

Fraile-Jurado, P., Rodríguez, E., Fernández, M., López, M., Torres, J. (2014). Estimación del comportamiento futuro del nivel del mar en las Islas Canarias a partir del análisis de registros recientes. Geographicalia, Vol. 66, pp. 79-98.

García-Herrera, R., Gimeno, L., Hernández, E., Prieto, R., Ribera, P. (2000) Reconstructing the North Atlantic atmospheric circulation in the 16th, 17th and 18th centuries from historical sources. Climate research, Vol. 14, N. 2, pp. 147-151.

García-Herrera, R., Gallego, D., Hernández, E., Gimeno, L., Ribera, P. (2001). Influence of the North Atlantic oscilation on the Canary Islands precipitation. Journal of Climate. Vol 14, pp. 3889-3903.

García-Herrera, R., Gallego, D., Hernández, E., Gimeno, L., Ribera, P. (2003). Precipitation trends in the Canary Islands. International Journal of Climatology. Vol. 23, pp. 235-241. doi: http://dx.doi.org/10.1002/joc.870 
Held, D., Hervey, A. Theros, M. (Eds). (2011). The Governance of Climate Change. Cambridge: Science, Economics and Ethics. Polity Press.

Máyer, P., Marzol, M. V. (2014). Daily precipitation concentration and the rainy spells in the Canary Islands: two risk factors. Boletín de la Asociación de geógrafos Españoles, Vol. 65, pp. 231-247.

Máyer, P., Marzol, M. V., Parreño, J.M. (2015). Tendencias de la precipitación en Canarias", Estudio, en aprovechamiento y gestión del agua en terrenos e islas volcánicas (II Workshop), Ma . C. Cabrera, T. Cruz-Fuentes, V. Mendoza-Grimón y $M^{a}$ P. Palacios-Díaz (Eds.), Instituto Geológico y Minero de España y Asociación Internacional de Hidrogeólogos, Las Palmas de Gran Canaria.

Martín, J.L., Bethencourt, J., Cuevas-Agulló, E. (2012). Asessment of Global Warming on the Island of Tenerife, Canary Islands (Spain). Trends in Minimum, Maximun, and Mean Temperatures since 1944. Climatic Change. Vol 112, N. 2, pp. 343-355. http://dx.doi.org/10.1007/s10584-012-0407-7

López, A., Dorta, P., Romero, C., Díaz, J. (2015). Movimientos de ladera en Canarias. El caso del Macizo de Anaga en el temporal de febrero de 2010. Análisis espacial y representación geográfica: innovación y aplicación. Universidad de ZaragozaAGE. pp. 1725-1734.

Luque, A., Martín, J., Dorta P., Mayer, P. (2014) Temperature Trends on Gran Canaria (Canary Islands). An Example of Global Warming over the Subtropical Northeastern Atlantic. Atmospheric and Climate Sciences. Vol. 4 N. 1, 2014, pp. 20-28. doi: http://dx.doi.org/10.4236/acs.2014.41003.

Olcina, J. (2012). Turismo y cambio climático: una actividad vulnerable que debe adaptarse. Investigaciones Turísticas. N. 4, pp. 1-34.

Semedo, A., Soares, P., Lima, D., Cardoso, R., Bernardino, M., Miranda, P. (2016). The impact of climate change on the global coastal low-level wind jets: EC-EARTH simulations. Global and Planetary Change, Vol. 137, pp. 88-106.

Tarife Méndez, R., Hernández Barrera, S., Gámiz-Fortis, S.R., Castro-Díez, Y. Esteban-Parra, Ma.J. (2012). Análisis de los extremos pluviométricos en las islas Canarias y su relación con el índice NAO. VIII Congreso Internacional AEC. Salamanca.

Tomé, R., Miranda, P., Brito de Azevedo, E., Teixeira, M. A. (2014). Climate Change in Small Islands. EGU General Assembly Conference Abstracts. Vol. 16, p. 9056. 